\title{
Behavior of Cohesive Powder in Rotating Drums
}

\author{
M. Wojtkowski*, O. I. Imole*, M. Ramaioli†, E. Chávez Montes** and S. Luding* \\ ${ }^{*}$ Multi-Scale Mechanics, Faculty of Engineering Technology, MESA+, \\ University of Twente, POBox 217, 7500 AE, Enschede, Netherlands. \\ ${ }^{\dagger}$ Nestlé Research Center, POBox 44, CH-1000 Lausanne 26, Switzerland \\ ${ }^{* *}$ Nestlé Product Technology Centre Orbe, Rte de Chavornay 3, CH-1350 Orbe, Switzerland
}

\begin{abstract}
We present experimental findings on the flowability and avalanching behavior of cohesive powders in a rotating drum. The main goal - beyond the scope of the current study - is to develop a method to understand and predict phenomena that precede the occurrences of events like avalanches and then to simulate this with the Discrete Element Method. In the present study, we focus on the characterization, classification, and description of the various events possible in cohesive powders - other than in non-cohesive particle systems - during rotation in a drum. Events are categorized based on their nature and we speculate on their relation to the micro-structure and properties of the powder.

As main result, we show that repeatable and consistent results can be obtained in the characterization of cohesive powders when angle-based (e.g. local surface and global center-of-mass) parameters are used. Different events can be distinguished, especially for strong cohesion, bulk shear sliding is often replaced by other events like slumping.
\end{abstract}

Keywords: Cohesive Powder, Avalanches, Cocoa, Rotating Drum, PARDEM

PACS: $45.70 . \mathrm{Ht}, 45.70 . \mathrm{Cc}, 81.05 . \mathrm{Rm}$

\section{INTRODUCTION}

Granular materials and powders are important raw materials for various commercial applications, e.g., in the agricultural, geotechnical, pharmaceutical, chemical and food processing industries [1, 2, 3]. An important challenge is the accurate characterization of the deformation and flow behavior of cohesive powders under static and dynamic conditions, as related to storage, handling and processing. There exists a large body of knowledge about experimental techniques to gain a better understanding of the behavior of these materials. When they are subjected to quasi-static deformations (in the macroscopic sense), one can measure the relationships between stress and strain at moderate and high compression stress levels [4]. For low stresses, close to the surface of the bulk material, since decades, laboratory tests in rotating drums have been used to understand the dynamic and shear behavior of particulate materials $[2,5,6,7]$. Various rotation regimes can be realized involving very slow, avalanching and very fast, centrifuging regimes. For cohesionless materials during rotation, the most evident observable property is the angle of the surface. This (continuous) "angle" at a given time frame in the drum can depend strongly on the side walls [8], which makes it more complicated to characterise such angles. Other avalanching or flowability tests [9] can provide more quantities that can be used to dynamically describe and classify the behavior of these materials. While experimental research to understand the dynamic behavior of various non-cohesive samples has been successful, many challenges remain for
TABLE 1. Material properties (size distribution $S_{d}$, particle density $P_{d}$ ) of the cocoa powder $C_{p}$ sample used, where the percentage indicates the fat-content.

\begin{tabular}{rrrrr}
\hline & & Unit & $C_{p}(12 \%)$ & $C_{p}(22 \%)$ \\
\hline \multirow{3}{*}{$S_{d}$} & $\left(x_{10}\right)$ & $\mu \mathrm{m}$ & 3.12 & 12.78 \\
& $\left(x_{50}\right)$ & $\mu \mathrm{m}$ & 8.68 & 24.23 \\
& $\left(x_{90}\right)$ & $\mu \mathrm{m}$ & 22.5 & 47.57 \\
$P_{d}$ & & {$\left[\mathrm{~kg} / \mathrm{m}^{3}\right]$} & 1509 & 1436 \\
\hline
\end{tabular}

sticky, cohesive powders like those commonly found in the food industry, see Ref. [10] and references therein. Here, we perform image analysis to characterize the behaviour of cohesive powders rotated in a drum. One goal is to find appropriate characterization parameters for cohesive powders. Those can, for example, be used for validating discrete element simulations, concerning the predictive quality of the flowability of cohesive materials. The well-established methodology for studying the flowability of cohesionless systems is applied and its limitations for strongly cohesive powder flow are highlighted.

\section{SAMPLE DESCRIPTION AND EXPERIMENTAL SET-UP}

The cohesive material studied is cocoa powder [3] with about $12 \%$ and $22 \%$ fat content. Material properties of 


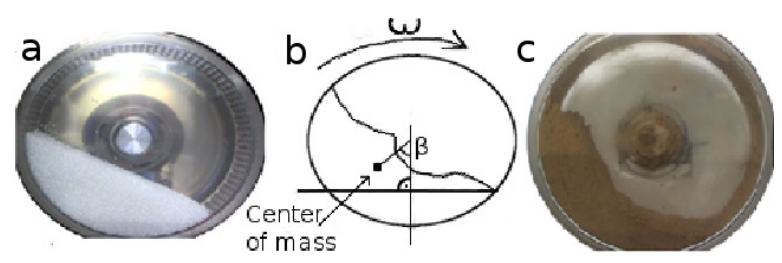

FIGURE 1. Snapshots and schematic representation of the powder surface angle and c.m.-angle for (a) glass beads (bright), (b) scheme of surface angle and rotation direction, and (c) cocoa powder with $12 \%$ fat content(dark).

the cocoa powder samples are shown in Table 1. The particle size distribution (PSD) is obtained by the "dry dispersion module" of the Malvern Mastersizer 2000 (Malvern Instruments Ltd., UK), while the particle density (not the bulk density) is obtained by helium pycnometry (Accupyc, Micromeritics, US).

The cohesive flow behavior is investigated with the AeroFlow tester (TSI Incorporated, USA), which rotates a shallow cylindrical plexiglass drum ( $125 \mathrm{~mm}$ diameter, $25 \mathrm{~mm}$ depth), containing the sample, around its horizontal axis, at a constant rate (angular velocity $\omega$ ) as shown in Fig. 1. When the inclination angle of the material (e.g. powder) surface becomes too great for its granular structure to support it, the powder collapses, which is referred to as an "event". To avoid wall sliding, an etched metal collar insert was placed around the drum's circumferential inner wall to increase the roughness and to obtain more regular, periodic events.

The time interval between events and their (relative) amplitudes are detected and recorded by a light fixture and photo-voltaic cell assembly positioned vertically in front and behind the drum, respectively. While the original, commercial set-up with a light sensor is capable of detecting big changes, it is impossible to distinguish events. Therefore, to obtain the profiles of the powder surface, an external camera (Logitech HD Pro, Logitech Intl SA) was mounted in front of the rotating drum and images were taken in regular intervals of $t_{i}=0.25$ seconds. Image analysis was performed and each event could be classified as discussed below, for more details see Ref. [10].

Samples with higher friction, non-sphericity and cohesivity are expected to have a higher angle of stability and possibly also a wider variability/range, i.e., larger times between successive events. Consistent results (with literature data) were obtained for glass beads of various sizes [10]. For cocoa powder, however, non-reproducible, irregular results are obtained due to the sticky nature of the powder and the limitations/disadvantages of the system leading to difficulties identifying the real avalanching events as opposed to undesirable events like sliding of the powder along the cylindrical wall of the drum or others. In the following, unless otherwise stated, results for the cocoa sample with $12 \%$ fat content are presented for the sake of brevity.

\section{POWDER CHARACTERIZATION}

In this section, we introduce the parameters used to characterize the state of the powder during experiments.

\section{Angle of surface and angle of stability}

For most non-cohesive samples the angle of surface is well defined (see Fig 1a). However, due to the irregular surface profile of cohesive samples, a global quantity that captures the position of the bulk sample relative to a fixed reference frame is desirable.

First, to obtain the (global) surface angle, the center of mass is computed. Every pixel in the snapshots of the drum (pixel size $\approx 6.25 \mathrm{~cm} / 360$ ) is analyzed along both vertical and horizontal directions. Using the pixels enables us to calculate the horizontal and vertical positions $-x$ and $y$, respectively - of the center of mass. Note that for this analysis, the powder layer sticking on the cylinder wall away from the bulk is not taken into account [10]. From this (at least for low filling height), the angle of surface is defined as $\beta=\operatorname{atan}\left(x_{c} / y_{c}\right)$, where $x_{c}$ and $y_{c}$ are the average values of pixels on which cocoa was detected, (see Fig. 1(b).

The (surface) angle for a powder in a rotating drum is thus defined as the angle between vertical and the line going through the center of material mass and the central point of the drum. From this, the average angle (of the surface profile) can then be computed as function of time, while the maximal angle, typically measured before the events, is referred to as "angle of stability". Even though no data are shown here this global quantity is expected to provide a distinction between avalanching and sliding of the bulk. The differences between the two event types, based on their respective angles of surface, will be studied in more details elsewhere, see Ref. [10].

\section{Events categories}

We classify the various events during the rotation of the cohesive samples into three categories based on the type of motion as schematically represented in Fig. 2 as follows: (a) Shear sliding - where the motion of the powder is due to shear failure within the sample, and the slip zone is far away from the circumferential wall of the drum. (b) Wall sliding - where powder motion is triggered by a shear band or slip zone along the circum- 


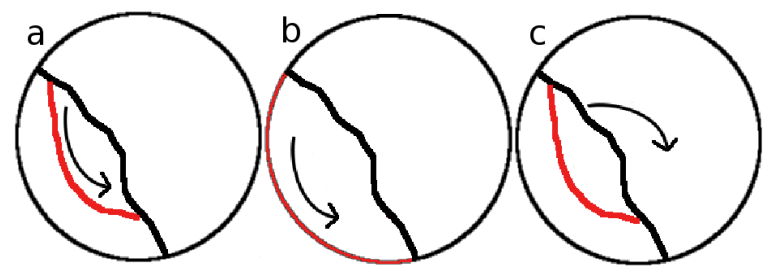

FIGURE 2. Schematic representation of event classification (a) shear sliding, (b) wall sliding and (c) slumping.

ferential length of the drum wall causing the material to slide - regardless of the metal collar inserted to attenuate this effect. (c) Slumping - a type of avalanche in which a mass of material breaks away along a curved surface and collapse downhill with some rotation in the drum rotation direction.

Note that events may overlap in time, so that the probability calculations for the events are not mutually exclusive. This leads to difficulties in categorization and investigating the relationships between different events. Also the non-linear shape of the surface of the material sometimes leads to errors in the image analysis which are, if detected, removed from the statistics.

During the experiment, crack-like defects appear within the samples. It has been shown in Ref. [11] that cracks are associated to granular slip events - and that these signals are precursors to shear failure in cohesive powders. The cracks can be identified by inserting voltage probes [11] within the sample. In this work, we identify cracks by simply observing fractures through the wall of the transparent drum. While this limits the generalization of our results, we expect that the findings presented hereafter will be valid and even clearer with the use of specialized instruments capable of probing into the material.

\section{RESULTS}

\section{Surface angle and angle of stability}

To characterize the cohesive powders in terms of the angle of the surface profile, we plot the probability histograms of angles for different volume filling fractions $(0.2,0.3$ and 0.4$)$ and two rotational speeds (1.2rpm and $0.3 \mathrm{rpm}$ ). Additionally, we plot the angle of stability [10], measured just before an event. As reference, we also show results for similar experiments performed on noncohesive $2 \mathrm{~mm}$ glass beads.

Figs. 3(a-c) contain the histograms of the stability angle at the high rotation speed of $1.2 \mathrm{rpm}$, while (d-f) contain the results for low speed $0.3 \mathrm{rpm}$. Reproducibility is confirmed by the collapse of the different realizations

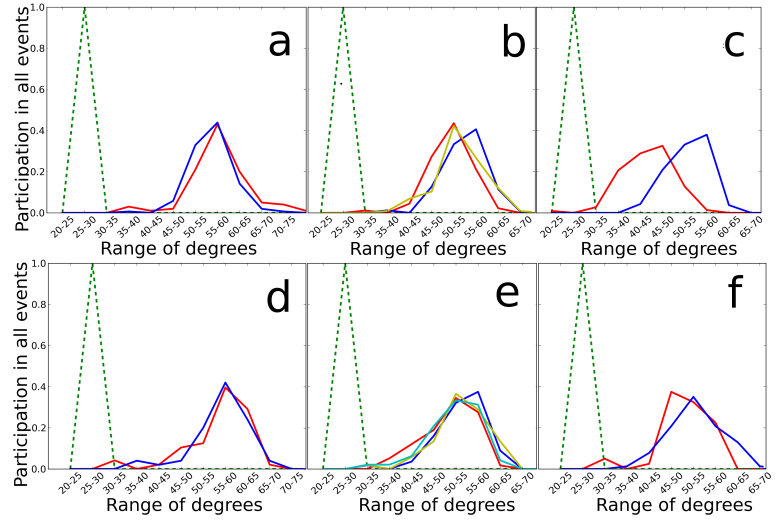

FIGURE 3. Probability histograms of angles of stability (before events) vs range of angles: a - volume of cocoa: $60 \mathrm{ml}$ speed of rotating drum: $1.2 \mathrm{rpm}, \mathrm{b}$ - volume: $90 \mathrm{ml}$, speed: 1.2 $\mathrm{rpm}, \mathrm{c}$ - volume: $120 \mathrm{ml}$ speed of rotating drum, $1.2 \mathrm{rpm}, \mathrm{d}$ - volume: $60 \mathrm{ml}$, speed: $0.3 \mathrm{rpm}$, e - volume: $90 \mathrm{ml}$, speed: $0.3 \mathrm{rpm}$, f - volume: $120 \mathrm{ml}$, speed: $0.3 \mathrm{rpm}$. The green dotted lines represent results for a glass bead experiment, with $2 \mathrm{~mm}$ diameter, as reference, the other colors (solid lines) different runs for cocoa powder.

on each other. Only for the largest filling fraction and higher speed, Fig. 3(c), the two experiments performed show different stability angle ranges from 30-55 and 4065 degrees, respectively. The same feature, but less pronounced, is also observed for the experiment run at lower speed shown in Fig. 3(f). Comparing both sets of data for various speeds, the stability angle variations are around 10 percent. However, the variation for cohesive powder is much higher than for the glass beads. In summary, as shown in Table 2, the cohesive cocoa powder displays a decreasing trend for the angle of stability with increasing filling fraction, while only for the largest fraction a significant decrease in angle is observed for the high speed data - for which more experiments and better statistics are required for confirmation.

\section{Events categories}

The main events during the tests on Cocoa $12 \%$ were shear sliding. The probability for this category is within $45-60 \%$ while the probability for slumping events is within $22-30 \%$. Increasing fat content of the cocoa sample to $22 \%$ leads to a significant reduction in shear sliding events. For this sample, we observe a 5-22\% probability of occurence of shear sliding events as against 75-95\% probability for slumping events. Note that events may overlap as such the probability calculations for the events are not mutually exclusive. 
TABLE 2. Values of average angles of surface $A_{s}$ in degrees and their standard deviation $S_{d}$ for different rotation speeds $\omega$ of experiments with glass beads $G_{b}$ and cocoa powder $C_{p}$ with $12 \%$ fat content with different volumes $V$ and speeds of the rotating drum $\omega$.

\begin{tabular}{rrrrrrrrr}
\hline & \multicolumn{1}{c}{$C_{p}, V=60 \mathrm{ml}$} & \multicolumn{2}{c}{$C_{p}, V=90 \mathrm{ml}$} & $C_{p}, V=120 \mathrm{ml}$ & \multicolumn{2}{c}{$G_{b}, V=90 \mathrm{ml}$} \\
\hline$\omega$ & $A_{s}$ & $S_{d}$ & $A_{s}$ & $S_{d}$ & $A_{s}$ & $S_{d}$ & $A_{s}$ & $S_{d}$ \\
\hline $1.2 \mathrm{rpm}$ & 57.8 & 5.5 & 53.1 & 4.8 & 48.6 & 6.8 & {$[-]$} & {$[-]$} \\
$0.3 \mathrm{rpm}$ & 56.2 & 6.6 & 52.6 & 5.9 & 52.1 & 5.9 & 27.9 & 0.6 \\
\hline
\end{tabular}

\section{Micro-Structure “Cracks”}

A comparison between the fraction of bulk solid transfer and crack propagation between sequential events (not shown) reveals a higher number of cracks in the less cohesive sample (with $12 \%$ fat content) compared to the highly cohesive sample (with $22 \%$ fat content). Fat content also has influence on the frequency of events. Increasing the fat content of the sample leads to a decrease in the frequency of events, in total. We note an approximate two-fold increase in the time between events from between $3-4.1$ secs for the $12 \%$ fat sample to times between 5.85 - 8 secs for the $22 \%$ sample A significantly higher number of cracks within of the $12 \%$ fat samples compared to $22 \%$ fat samples is possibly due to the finer texture of the grains and its lower fat content. For cocoa ( $22 \%$ fat), the sample is more solid and cracks rarely form during the events.

We now speculate about an interpretation: Crack formation is related to shear sliding events and thus, since slumping events are more pronounced in samples with higher fat content, those display lesser of the cracks. A significant number of shear sliding events occur alongside with crack formation in the material. This implies a correlation between shear sliding and cracks, even though we were not able to observe all cracks inside the material - only those which were visible on front wall. A complementary quantification method for the number of shear sliding events associated with crack formation, as measured in Ref. [11] using non-contact voltage probes, will be the subject of future studies.

\section{CONCLUSION}

An experimental analysis of the behavior of cohesive powders in a rotating drum was presented. It has been shown that while the accurate characterization of cohesive powders remains a challenging problem, significant quantitative information can be obtained by image analysis, considering the surface angle as a complement to other quantities like the time between events and the scatter- or attractor-plots, as based on the simple lightsensor device - which works well for non-cohesive reference samples but not for cohesive materials. Repeatability and consistency of the results is established for low drum filling fractions. We found that slumping events predominate at high cohesion (higher fat content), while shear sliding is dominant at low cohesion (low fat content).

\section{ACKNOWLEDGEMENTS}

Helpful discussions with N. Kumar and V. Magnanimo are acknowledged as well as the support of the European Community under the Marie Curie Initial Training Network PARDEM (www.pardem.eu).

\section{REFERENCES}

1. B. H. Kaye, Powder mixing, vol. 10, Springer, 1997.

2. B. H. Kaye, J. Gratton-Liimatainen, and N. Faddis, Particle \& particle systems characterization 12, 232-236 (1995).

3. G. Wolf, Nahrung 25, 606 (1981).

4. J. Schwedes, Granular Matter 5, 1-43 (2003).

5. R. Brewster, G. S. Grest, and A. J. Levine, Physical Review E 79, 011305+ (2009).

6. C. M. Dury, and G. H. Ristow, Phys. Rev. E 57, 4491-4497 (1998).

7. J. Mellmann, Powder Technology 118, 251-270 (2001).

8. N. Taberlet, P. Richard, and E. John Hinch, Phys. Rev. E 73, 050301 (2006).

9. K. Thalberg, D. Lindholm, and A. Axelsson, Powder technology 146, 206-213 (2004).

10. M. B. Wojtkowski, O. I. Imole, M. Ramaioli, E. Chavez, and S. Luding, In preparation (2013).

11. T. Shinbrot, N. H. Kim, and N. N. Thyagu, Proceedings of the National Academy of Sciences 109, 10806-10810 (2012), ISSN 1091-6490. 